\title{
A comparison of an explicit and an implicit transient strain formulation for concrete in fire
}

\author{
DAVID LANGE and ROBERT JANSSON \\ SP Technical Research Institute of Sweden \\ Department of Fire Technology \\ Box 857 \\ SE-501 15 Borås
}

\begin{abstract}
This paper reviews the phenomena of concrete behavior which is captured in the material data of the Eurocode and reviews additional phenomena which may be included to improve results in certain cases. As a case study, a series of tests which were conducted by Anderberg and Thelandersson in the 1970's in Sweden on concrete samples are studied using first of all the temperature dependent material model which is used in the Eurocodes. The impact of load induced thermal strain is discussed through comparison of the implicit model which is used in the Eurocode and a simple explicit model based on the test results.
\end{abstract}

KEYWORDS: Protection of concrete, modeling, material behavior

\section{NOMENCLATURE LISTING}

\begin{tabular}{llll}
$T$ & Temperature & \multicolumn{2}{c}{ subscripts } \\
$t$ & time & $t h$ & thermal \\
$k$ & material property reduction factor & $\sigma$ & stress related \\
$K$ & constant (dependant upon mix) & $c r$ & creep \\
$f$ & strength & $\theta$ & temperature \\
\multicolumn{2}{|l}{ Greek } & $c$ & compressive \\
$\varepsilon \quad$ strain & $u$ & ulitmate \\
$\sigma$ & stress & & \\
$\tilde{\sigma}$ & stress history & &
\end{tabular}

\section{INTRODUCTION}

\section{Components of strain in concrete}

For advanced calculation methods, Eurocode 2 describes the total strain in concrete in terms of the sum of 4 components [1], the thermal strain, $\varepsilon_{t h}$; the instantaneous stress-dependent strain, $\varepsilon_{\sigma}$; the creep strain, $\varepsilon_{c}$; and the transient state, or transient, strain, $\varepsilon_{t r}$ :

$$
\varepsilon=\varepsilon_{t h}(T)+\varepsilon_{\sigma}(\sigma, T)+\varepsilon_{c r}(\sigma, T, t)+\varepsilon_{t r}(\sigma, T)
$$

Where the thermal strain is dependent upon the temperature $T$; the mechanical strain is dependent upon the current stress state, $\sigma$, and the temperature; the creep strain is dependent upon the stress, temperature and time, $t$; and the transient strain which is dependent upon the stress and the temperature. It is also acknowledged that the stress related strain is also dependent upon the stress history, $\tilde{\sigma}$, e.g. [2]. Therefore the stress-dependent strain term may in fact be written $\varepsilon_{\sigma}(\tilde{\sigma}, \sigma, T)$. Because of the time scale required for creep strains to develop, these are often omitted from structural fire calculations. This reduces the total strain as defined above to be a function of the free thermal expansion strains, the stress induced strains and the transient strain. 


\section{Transient strain or LITS}

Transient strain, or transient thermal strain, and LITS are terms which are often used interchangeably. It is comprised variously of a number of components depending upon the definition used by the author, including, e.g. basic creep strain, transient creep, shrinkage. Law presented a review of the mechanisms which contribute to LITS in his thesis [3]. His definition of LITS was: "LITS is the difference between the free thermal expansion of concrete and the thermal expansion when the same concrete is heated under a level of pre-stress". This is in general agreement with Anderberg and Thelandersson's definition of transient strain which is 'that part of the total strain obtained in stressed concrete under heating that cannot be accounted for otherwise'[4], i.e. from the other components of the total strain in concrete discussed above. It is important to note however that LITS as defined by Khoury also includes the basic creep, as well as the temperature induced change in elastic strain and a transient creep term which may also be easily confused with the term transient strain [5].

These two definitions are illustrated in Fig. 1. To avoid confusion this article is concerned with a discussion of the impact of transient strain on the response of heated concrete structures.

In the Eurocode, specifically EN1992-1-2:2004 [1], transient strain is not explicitly dealt with and its effects are accounted for in heated concrete implicitly. That is to say that the strain parameters required to define the stress-strain curve in fire are artificially increased with increasing temperature beyond their 'actual' values. This has the effect of decreasing the tangent modulus by a fixed amount at each temperature increment, allowing some equivalent transient strain to develop at constant load levels with increasing temperature. This formulation of transient strain however limits the amount of transient strain to a maximum, according to the adjustment that was made when the material properties were defined. Historically this formulation has been shown to give good results when comparing the results of calculations of the response of concrete elements in fire to the results of fire tests. Anderberg however suggests that this is simply 'luck' that failure is predicted using the implicit material model [6]. Nevertheless, for most applications involving reinforced concrete elements, where the compressive region is at the top of the element loaded in flexure the effective compressive preload in any concrete which will be heated is negligible so this is perhaps not surprising. Further recent work has however highlighted that there are a number of cases whereby the role of the implicit transient strain model in the Eurocode is not necessarily suitable, most importantly where high levels of load are present or where there is some cyclic nature to the loading, for example during cooling following a fire.

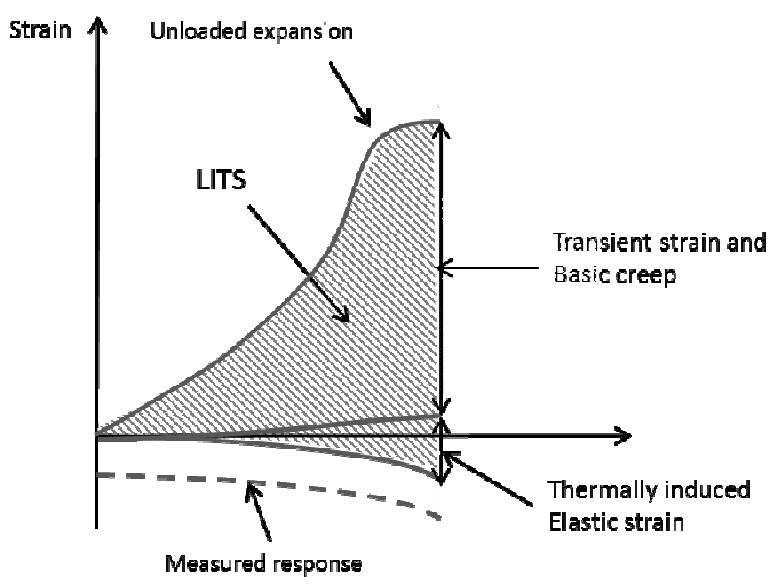

Fig.1. LITS is the difference between free thermal expansion of concrete and the expansion under a level of pre-stress and includes transient creep, basic creep and elastic strain, adapted, transient strain is the difference between the free thermal expansion of concrete and the thermal expansion when the same concrete is heated under a level of pre-stress which does not include creep strain or stress induced strain

Gernay and Franssen developed a formulation of the Eurocode 2 material model including an explicit transient strain component [7,8]. In their study they reviewed the stress development at various points 
within a loaded concrete cylinder subject to heating and concluded that transient strain plays a significant role in the behavior of loaded concrete specimens, especially during cooling. They concluded in their example that the residual axial load sustained by a column at the end of a fire comprising a heating and a cooling phase could be overestimated by up to $25 \%$ of the initial load if an implicit transient creep strain model is used

Huang et al. presented an implementation of explicit transient strain in the Vulcan finite element software and studied the impact of transient strain on the buckling resistance of RC columns in fire [9]. It was found that the effect of including a formulation for transient strain in their model was significant and resulted in significantly reduced buckling times in fire.

This article will review the impact of transient strain which is illustrated by a series of tests from the literature before reviewing the ability of the Eurocodes implicit transient strain formulation in reproducing the results from these tests. Then, using an explicit formulation of transient strain along with additional material data from the literature the model will be shown to correlate better with the results of the tests. Finally, a comparison is made between the two formulations for the analysis of a prestressed concrete section exposed to fire.

\section{TRANSIENT STRAIN}

\section{Tests loaded during heating}

Anderberg and Thelandersson conducted a series of tests, reported in 1976 [4], which illustrate the effect of transient strain on the response of concrete cylinders under various levels of preloading. In total 93 tests were performed under various loading conditions and different heating rates. The test specimens comprised concrete cylinders of diameter $75 \mathrm{~mm}$ and height $150 \mathrm{~mm}$ and with a circular hole of diameter $10 \mathrm{~mm}$ in their center running the entire height of the cylinders.

Of the 93 tests which were conducted, only 5 are discussed in this article. These specific tests are tests A5, A6, A7, A8 and A9. These represent a selection from the series of tests which were conducted under a constant load which was applied before heating started and under a constant heating rate of $5^{\circ} \mathrm{C}$ per minute from the ambient temperature up to $800^{\circ} \mathrm{C}$. All samples were subject to the same treatment before the experiement (they were stored in a controlled environment of $20^{\circ} \mathrm{C}$ at $65 \%$ relative humidity for between 21 and 26 weeks before the test). A summary of the concrete strength and the loading is shown in Table 1 . The stress level of the concrete is based on the ambient strength of the concrete which is assumed to be $75 \%$ of the cube strength.

Table 1. Summary of test specimens, adapted from [4].

\begin{tabular}{|r|r|r|}
\hline Specimen number & Stress \% & Cube strength (MPa) \\
\hline A5 & 0 & 55.1 \\
\hline A6 & 22.5 & 58.4 \\
\hline A7 & 35 & 52.4 \\
\hline A8 & 45 & 64 \\
\hline A9 & 67.5 & 58.4 \\
\hline
\end{tabular}

Figure 2 shows the strain in the vertical axis of the cylinders during the tests. These results have had the initial mechanical stress related strain, $\varepsilon_{\sigma}$, removed from the series shown. At $0 \%$ load, the strain equals the unrestrained thermal expansion of the material. At $22.5 \%$ load level the total strain is affected by the transient strain and the net strain of the specimen before failure under the applied load is significantly lower than the free thermal expansion. With increasing load level the impact of transient strain increases and between $35 \%$ and $45 \%$ load level the contribution of transient strain in fact results in a net shrinkage of the concrete under thermal loading and before failure of the specimen as a result of reduction in the strength of the specimens due to temperature. 


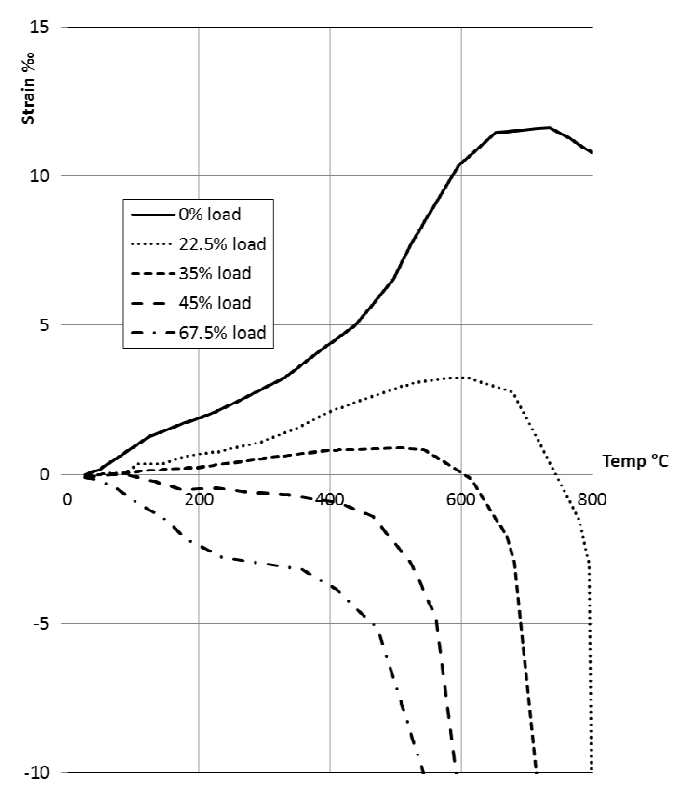

Fig. 2. Longitudinal strain in heated concrete under varying load levels, adapted from [4].

\section{INITIAL MODELLING}

\section{EN 1992 concrete material parameters}

Figure 3 shows the reduction factor for the ultimate strength of concrete in EN 1992-1-2:2004 [1] as well as the yield and ultimate strain of concrete with temperature. These three parameters are used to define the stress-strain relationship of concrete at high temperature using equation (2) in the ascending region.

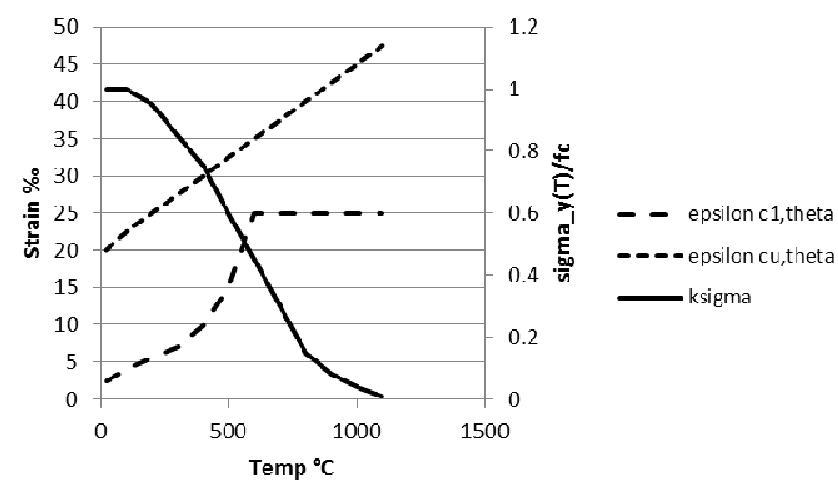

Fig. 3. Reduction factor for concrete strength at high temperature and the yield and ultimate strain of concrete including an implicit term for transient strain, adapted from [1]

$$
\sigma(\theta)=\frac{3 f_{c, \theta}}{\varepsilon_{c 1, \theta}\left(2+\left(\frac{\varepsilon}{\varepsilon_{c 1, \theta}}\right)^{3}\right)}
$$

In the descending region, which is included in the Eurocode for numerical modeling purposes the stress strain relationship reduces from the peak strength of the material at a strain $\varepsilon_{c l, \theta}$ to zero strength at a strain of $\varepsilon_{c u, \theta}$. No specific guidance is given about the shape of the descending branch and it may be either linear or non-linear according to the engineer's discretion. 


\section{Initial modeling results}

In order to evaluate the ability of the material parameters in the Eurocode in predicting the strain response of the cylinders the material parameters described above were implemented in finite element models of the tests conducted by Anderberg and Thelandersson. The concrete was heated at $5{ }^{\circ} \mathrm{C} / \mathrm{min}$, as in the tests and the calculations were carried out using the implicit formulation of the Eurocodes using the appropriate parameters for the material definition based on the reported strength of the concrete.

In order to try to remove mesh dependency upon the results, the mesh used was an unstructured mesh comprising tetrahedral elements.

Fig. 4 shows the strain in the concrete cylinders as determined using the material properties from EN 1992:1-2:2004 [1] in the numerical model. These properties have an implicit transient strain formulation. In the figure, the darker lines indicate the results of the calculations. The grey lines indicate the results of the tests performed by Anderberg and Thelandersson as described above. At $0 \%$ load, the unloaded specimens follow the free thermal expansion curve. The free thermal expansion of concrete as given in the Eurocode follows generally the same trend as the free thermal expansion of the concrete which was used in the tests, although the maximum value is higher (the Eurocode concrete material properties approach 15\%o peak thermal expansion as opposed to around $12 \%$ as was observed in the tests). At $22.5 \%$ load level, the Eurocode material properties result in a strain under heating which closely follows the test results - peak net strain is within about $2 \%$ of that observed in the test. However increasing the load level results in a smaller contribution from the transient strain to the net strain when transient strain is included in the model implicitly and the calculated results deviate quite considerably from the test results. Indeed, when the transient strain is such that the net strain observed in the tests was negative the implicit transient strain formulation is not able to follow this and the calculated net strain simply becomes increasingly small and it is an increase in the stress related strain which results in a negative net strain. With regards to the mechanical response of the specimens during the calculations it can be seen that onset of runaway of the specimens occurs at a slightly lower temperature than is reported from the tests.

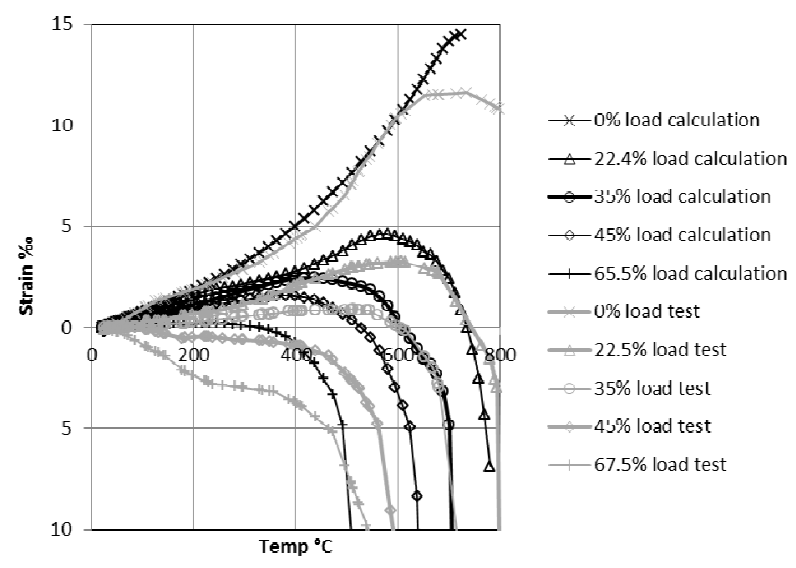

Fig. 4. Strain in the test cylinders calculated using Eurocode 2 concrete parameters and implicit transient strain

\section{Stress distribution in the cylinders}

It is important to note that the stress distribution in the cylinders under different load levels varies, with the maximum tensile stresses forming on a horizontal slice through the specimen.

With no load, cracks develop in the cement paste around the aggregate as the cement paste shrinks at higher temperature when the aggregate is still expanding. A preload during testing partially closes this crack development in the direction orthogonal to the compressive load and at high load ratios larger cracks are created parallel to the loaded direction. Transient strain may therefore be explained by means of, with the help of load, not opening up the cracks developed by the difference in thermal expansion of the aggregates vs. the cement paste, microcracks in the concrete continue to form orthogonal to compressive loading. 
For example, under $0 \%$ load in the tests discussed, a compressive ring forms around the perimeter of the specimen. This compressive ring forms as a result of expansion of the circumferential 'layer' of concrete which is restrained by the inner region of concrete. This causes tensile stresses to develop radially in the central region of the concrete. No closure of the cracks occurs within the specimen except for a small amount in this radially stressed region and minimally throughout as a result of strain compatibility within the sample.

Under increasing load however, compressive stresses run axially through the specimen, partially closing the cracks which open as a result of differential expansion of the paste and the aggregate - the level of closure is dependent upon the load level. However there is little to no change in the trend of the tensile stresses. As with the specimen under $0 \%$ loading, these are also concentrated radially throughout the section of the specimen. Transient strain behavior is therefore most likely not isotropic.

\section{EXPLICIT TRANSIENT STRAIN FORMULATION}

\section{Concrete parameters with explicit consideration of transient strain}

Examples of strain rate controlled tests are reported by Schneider [10] and are used for the development of a set of variables, $\mathrm{f}_{\mathrm{c}, \theta} / \mathrm{f}_{\mathrm{ck}}, \varepsilon_{\mathrm{c} 1, \theta}$ and $\varepsilon_{\mathrm{cul} 1, \theta}$, corresponding to the parameters required in Equation 2 . These results are presented since they do not have any transient strain term added. The stress strain relationship resulting from these tests is reproduced in Fig. 7. The variables arising from these stress-strain curves at different temperatures are shown in Fig. 8. Two lines are shown for $\varepsilon_{\mathrm{cul} 1, \theta}$. The first one corresponds with the actual measured value from the tests where there is a peak at $350{ }^{\circ} \mathrm{C}$. This is also visible in the data reproduced in Fig. 7 where the ultimate strain at $350^{\circ} \mathrm{C}$ is considerably higher than the ultimate strain at $150^{\circ} \mathrm{C}$ or $450^{\circ} \mathrm{C}$. Since this peak deviates from the trend of the values for $\varepsilon_{\mathrm{cul} 1, \theta}$, a modification to the ultimate strain is proposed whereby the peak is removed and the ultimate strain is set to $5 \%$ o above the crushing strain. These strains are significantly lower than the corresponding strains as given in the Eurocode parameters for the stress-strain relationship of normal weight concrete at elevated temperatures (Fig. 3).

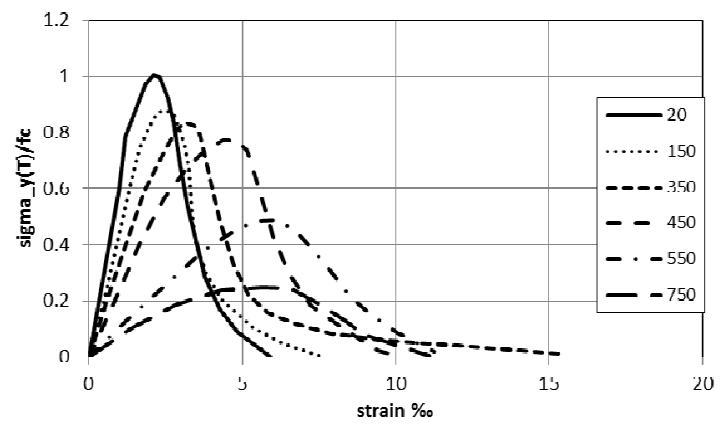

Fig. 7. Stress - strain curves of concrete at elevated temperatures under strain rate controlled steady state testing [10].

Also of note is that the reduction in yield strength of concrete observed by Schneider is not as severe at low temperatures as the reduction which is suggested in the Eurocode. Yield strength is largely constant (losing only $10 \%$ ) up to around $400^{\circ} \mathrm{C}$, before it reduces to $40 \%$ of its ambient strength between 400 and $600^{\circ} \mathrm{C}$. For modeling purposes, the factor $\mathrm{f}_{\mathrm{c}, \theta} / \mathrm{f}_{\mathrm{ck}}$ was extrapolated and reduced linearly between $25 \%$ at $750^{\circ} \mathrm{C}$ and $1 \%$ at $1100^{\circ} \mathrm{C}$, this final value corresponding with the lowest value specified in Eurocode 2.

The parameters of concrete material strength which are interpolated above agree generally well with those found in literature for specimens unloaded during heating. For example, Bazant and Kaplan [11] provide a review of a number of tests on concrete. In the tests which are discussed, unstressed concrete retains 80 to $100 \%$ of the compressive strength up to $400^{\circ} \mathrm{C}$, after which it experiences a rapid reduction with increasing temperature. Schneider also reviewed a number of other test results in the literature [12] which show a similar trend in the compressive strength of heated concrete. Further, Schneider summarized the ultimate strain of various concrete mixes comprising different aggregate types, and these were found to vary between $2 \% \circ$ and $3 \%$ at ambient temperature and between $6 \%$ and $9 \%$ at $700^{\circ} \mathrm{C}$. 
In comparison with the compressive strength variation with temperature and the ultimate strain reported by Anderberg and Thlandersson for their loaded samples [4], the values reproduced above are also within a similar range. However Anderberg and Thelandersson do not report any compressive strength data for the samples relating to the tests described above between ambient temperature and $400^{\circ} \mathrm{C}$. They also only report one data point from their tests at $45 \%$ loading. Their reported values of ultimate strain during heating are between $\sim 3 \%$ at ambient temperature and $8 \%$ at $800^{\circ} \mathrm{C}$ for samples unloaded during heating; however the ultimate strain value for the sample loaded during heating is relatively constant between $200^{\circ} \mathrm{C}$ and $700^{\circ} \mathrm{C}$, with a range of $\sim 2.5 \%$ and $\sim 4 \%$. In order to illustrate the effect of including transient strain explicitly in the concrete model, the results reported by Schneider will therefore be used as representative of the behavior of concrete not including a transient strain term.

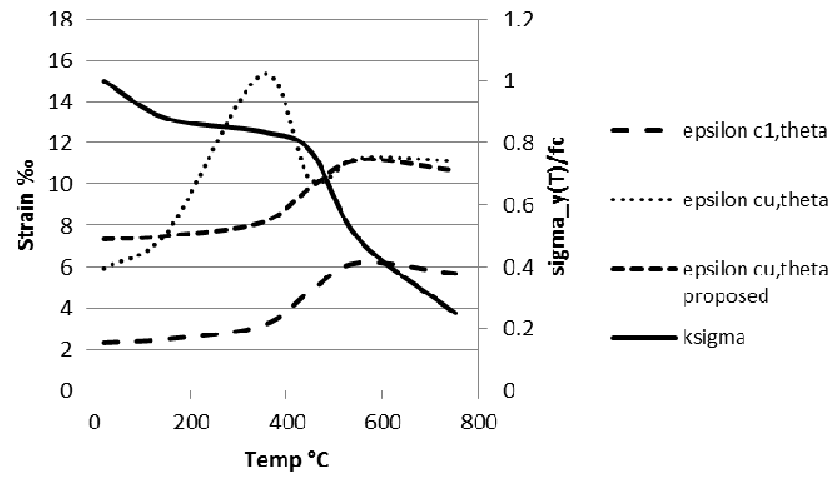

Fig. 8. Relationship between ultimate strength of concrete at elevated temperature under instantaneous loading, and the yield and ultimate strain of concrete not including transient strain

Clearly these values of ultimate and crushing strain are significantly lower than those given in Eurocode 2 and the variation of strain with temperature is significantly lower. These factors will have a significant impact on the tangent modulus of concrete. EN 1992-1-1: 2004 [13] defines an approximate initial tangent modulus through the tangent between the angle of the x-axis of the stress strain diagram and the stressstrain curve at a value corresponding to $0.4 f_{c m}$; where $f_{c m}$ is $80 \%$ of the crushing strength of concrete. Alternatively, the derivative of equation (2) may be used to obtain the initial tangent modulus. Because of the smaller variation in crushing strain with temperature using the parameters described omitting transient strain then the variation in tangent modulus is also less. A comparison between the initial tangent modulus based on the Eurocode 2 parameters and the parameters shown in fig. 8 is shown in fig. 9. As with the yield strength, the tangent modulus for the material parameters not including transient strain retains 80 to $90 \%$ of its ambient value up until around $400^{\circ} \mathrm{C}$, at which point it reduces almost linearly toward $0 \%$ at $1100^{\circ} \mathrm{C}$.

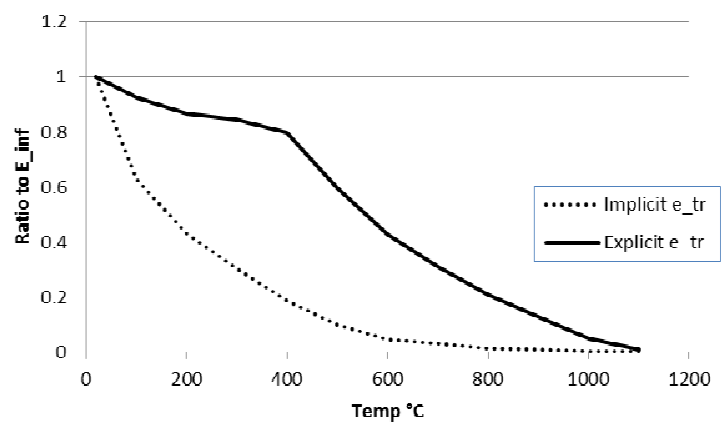

Fig. 9. Relationship between the initial tangent modulus of concrete and temperature.

With the transient strain term not included in the material parameters, it is necessary to include it explicitly in accordance with equation (1). There are several different formulations for transient strain proposed by different authors including e.g. Khoury et al., who developed a complete mathematical model for concrete comprising multiple phases which is capable of predicting, amongst others, transient strain phenomena [14]. Schneider also developed a concrete material model which included transient strain phenomena [15]. 
Anderberg and Thelandersson developed a model for transient strain [4]. As with Khoury's model, the transient strain is related to the load level. In this case it is related by means of a curve fit of a factor, $K$, to the experimental data discussed above. This factor varies with concrete mix, however for the data shown $K=2.35$. The transient strain term is given by the following equation:

$\varepsilon_{t r}=-K \frac{\sigma}{\sigma_{u}} \varepsilon_{t h}$

It was noted that at temperatures over $550^{\circ} \mathrm{C}$ the correlation is not so good. For these temperatures, the following modification has been proposed [16]:

$$
\Delta \varepsilon_{t r}=0.1 \times 10^{-3} \Delta T \frac{\sigma}{\sigma_{u}}
$$

It is not the objective of this article to review the different material model definitions for concrete which include transient strain explicitly. Rather to compare the results of including an explicit formulation of transient strain with an implicit formulation. Therefore the original model proposed by Anderberg and Thelandersson, using equation (3), will be used throughout the remainder of this article. This formulation was also shown to give good results in comparison with their own experiments when it was first reported [4].

\section{Implementation of explicit transient strain material model}

Using the finite element software Abaqus, transient strain is implemented in the finite element analysis as a modification to the thermal expansion coefficient, $\alpha$. Since the effect is directionally dependent, i.e. stresses in one direction will not induce transient strain in other directions, orthogonal thermal expansion coefficients are defined. These orthogonal thermal expansion coefficients are dependent upon three additional field variables implemented in the software. The field variable corresponds to the value of equation (3) at each integration point and for each local material axis, calculated at the current increment. The maximum value of each field variable up until the current increment is recorded in additional state variables at the integration points and the field variable is not allowed to reduce in value in the event of unloading, thus imparting some load history dependency upon the model. This means of implementing transient strain in the model was adopted since transient strain is defined, as described above, as the difference between the free thermal expansion and the net expansion as a result of heating under a confining force and it does not result in any additional mechanical stresses developing within the material. There is no suggestion that transient strain and thermal expansion are in any way similar mechanisms however the transient strain may be used to modify the net expansion since no additional stresses develop directly as a result of the transient strain.

\section{FINITE ELEMENT MODELLING}

\section{Strain in the cylinders}

In order to compare the strain response of the explicit and the implicit transient strain the finite element calculations were repeated using the parameters derived from the instantaneous loaded tests using the transient strain formulation proposed by Anderberg and Thelandersson. As above, the study was carried out using an unstructured mesh comprised of first order quadratic elements.

Figure 10 shows the same results for the calculations which were performed using the explicit transient strain formulation incorporated into the numerical model as described above. In this instance, the free thermal expansion has also been adjusted to reflect the free expansion observed in the tests. As expected therefore, the test and calculation with $0 \%$ loading agree well with each other. At $22.5 \%$ load level, the strain also agrees well, with closer approximation of the net strain under thermal loading than in the calculation performed using the Eurocode model. With increasing load level, however, there is considerably better agreement using the explicit transient strain formulation than when the Eurocode 
material model is used. The different calculations follow their respective tests far more closely and the net strain decreases and returns a negative value at higher load levels. However, as the material properties which were used to estimate the parameters required for the concrete model were based on the results of tests reported by Schneider as described above the concrete material failure does not agree and in fact onset of runaway occurs at a slightly higher temperature than in the test. Nevertheless it can be seen that the inclusion of the explicit transient strain formulation significantly improves the phenomenological agreement with experimental results.

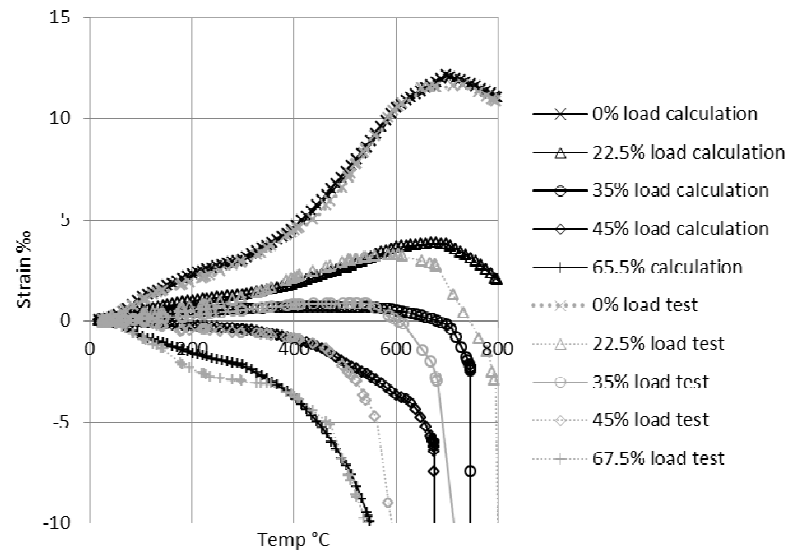

Fig. 10. Strain in the test cylinders calculated using the explicit transient strain formulation

Recalling that the transient strain is accounted for in the Eurocode material parameters by means of adding a fixed strain to the strain parameters which define the stress-strain behavior it can be seen that the amount of transient strain is effectively fixed at high load levels. That is to say that although it is stress-dependent (since increasing the stress will move the material further along the stress-strain curve) the amount of transient strain available will not increase with higher loads. This is contrary to what may be seen in Fig. 2 above, and is evidenced by Figures 4 and 10 where increasing the load level when the Eurocode material model is used does not result in equivalent net strains to those observed in the tests, but including the explicit formulation does.

\section{IMPACT ON STRUCTURAL BEHAVIOUR}

In order to illustrate the application of this transient strain formulation on structural behavior a simple example is analysed using the finite element method. The element chosen is a bonded prestressed concrete element of section size $400 \mathrm{~mm} \times 1200 \mathrm{~mm}$. The concrete has a characteristic strength of $60 \mathrm{~N} / \mathrm{mm} 2$. Three tendons, diameter $37.4 \mathrm{~mm}$, are evenly distributed at a depth of $870 \mathrm{~mm}$ within the section and have characteristic strength $1700 \mathrm{~N} / \mathrm{mm} 2$, modulus of elasticity of $200000 \mathrm{~N} / \mathrm{mm} 2$ and an initial yield of 1220 $\mathrm{N} / \mathrm{mm} 2$. The tendons are prestressed to $910 \mathrm{~N} / \mathrm{mm} 2$.

The span of the element is $10 \mathrm{~m}$, it is simply supported and is subject to a uniform distributed load resulting in a peak moment of $2500 \mathrm{kNm}$. To prevent issues with transfer of the prestress within the model at the ends of the beam, a 50mm length at the end is comprised of a stiff linear elastic material. The degrees of freedom in the solid elements comprising the tendons are tied to corresponding nodes within the concrete. A single axis of symmetry is employed in the model at midspan. It is exposed on three sides to an ISO fire of duration 1 hour, defined via a radiation temperature and a gas temperature which follow the standard fire curve, a surface emissivity of 0.8 and convective heat transfer coefficient of $25 \mathrm{~W} / \mathrm{m}^{2} \mathrm{~K}$. The analysis was carried out using both the implicit transient strain formulation for concrete which is in the Eurocode and the explicit formulation developed by Anderberg and Thelandersson discussed above.

Although simplistic, this example may be considered to be representative of a precast element which is ungrouted within a structural frame.

The effects of heating on a simply supported element have been well documented [15]. In the case of a simply supported prestressed element; during heating of the concrete expansion of the concrete forces the element to 'push' against the effective restraint which is provided by the tendons. This causes an increase in stress and strain in the tendons. Continued heating of the element then results in a temperature rise and 
thermal expansion in the tendons which results in a reduction in the stress by relieving a part of the elastic strain in the tendons without a reduction in the overall strain. This occurs first at the outermost tendons and is then followed by the inner tendons. This effect can be seen in Figures 11 and 12 which show the stress and the strain in the tendons.

The impact on the tendons of the change in the transient strain formulation within the concrete is also visible in Figures 11 and 12. In this case, the Eurocode model has a lower apparent transient strain component, resulting in a higher overall stress in the tendons, despite the effect of thermal expansion of the steel upon heating. A higher net strain in the tendons is also observable.

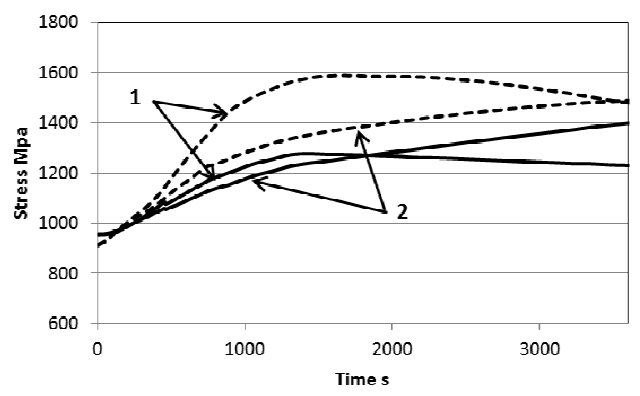

Fig. 11. Stress in the prestressing tendons - dashed lines indicate results when using the EC concrete material properties and the solid line indicates results obtained when using the explicit transient strain formulation. The outer tendons are labeled ' 1 ' and the middle tendon is labeled ' 2 '

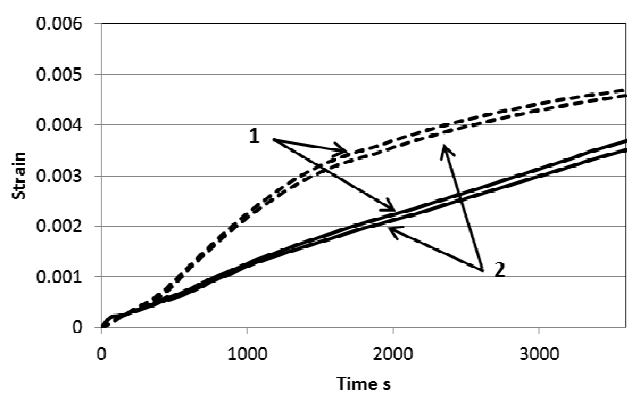

Fig. 12. Strain in the prestressing tendons - dashed lines indicate results when using the EC concrete material properties and the solid line indicates results obtained when using the explicit transient strain formulation. The outer tendons are labeled ' 1 ' and the middle tendon is labeled ' 2 '

This change in the prestress and the lower strain in the tendons also has an impact on the overall behavior of the element, with a marked increase in the deflection of the concrete element in fire as a result of the lower stress in the tendons when using the explicit transient strain formulation over the eurocode model, Fig. 13.

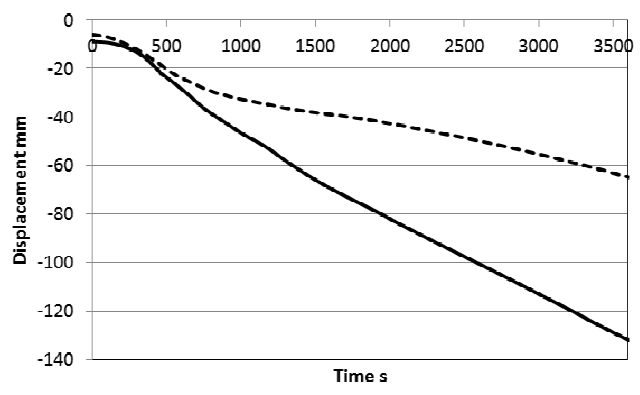

Fig. 13. Deflection of the prestressed concrete element - the dashed line indicates results when using the EC concrete material properties and the solid line indicates the result obtained when using the explicit transient strain formulation 


\section{CONCLUSIONS}

This paper has presented a short study comparing the results of different models when using the implicit eurocode model for transient strain and an explicit formulation from the literature.

It has been demonstrated that the impact of the material properties which are used for concrete in a numerical model can be large when considering the phenomenological behaviour. When comparing the results of modeling of tests from literature using the material properties from the Eurocode large differences were found between the modeling and the experiments. These result from the implicit formulation for transient strain which is included in the Eurocode concrete model, where the maximum possible transient strain is effectively fixed at some value depending upon the temperature. Conversely, when the same analyses are carried out including an explicit stress dependent formulation of transient strain which is not limited to a maximum value, the phenomenological behavior was found to agree much closer with the results of the tests. This is despite the fact that the concrete mechanical material properties which were used in the analyses did not correspond with the material which was used in the tests to which the analyses were compared. This shortcoming could be addressed in future work.

Finally, the paper compared the results of a simple analysis of a prestressed concrete element exposed to fire with material properties comprising an explicit and an implicit transient strain formulation. Significant differences were found in the global response of the element, based upon the displacement. These significant differences most likely arise from relatively small differences in the interaction between the tendons and the concrete beam between the two material formulations. No assertion is made with regards to the relative failure times of the element when analysed using the two formulations, and no comparison is made with the results of testing.

In summary, it has been shown that the implicit transient strain formulation in the Eurocodes performs well where the compressive loading of heated concrete is relatively small. With increased compressive loading, the implicit formulation does not exhibit the same phenomenological result as an explicit formulation.

\section{ACKNOWLEDGEMENTS}

The authors are grateful to the Swedish civil contingencies agency (MSB) for their financial support of this work as part of the Safe Multibygg project.

\section{REFERENCES}

[1] EN 1992-1-2:2004 Design of concrete structures, Part 1-2: General rules - Structural fire design

[2] Schneider, U.; Horvath, J.; Behaviour of ordinary concrete at high temperatures; Research reports of Vienna University of Technology, Institute of Building Materials, Building Physics and Fire Protection Vol. 9, Vienna, 2003.

[3] Law, A.; The assessment and response of concrete structures subject to fire; PhD thesis; The University of Edinburgh 2010

[4] Anderberg, Y.; Thelandersson, S.; Stress and deformation characteristics of concrete at high temperatures 2. Experimental investigation and material behavior model; Lund institute of technology, 1976

[5] Khoury, G.; Fire and concrete: from materials behaviour to application; International workshop on fire design of concrete structures from materials modelling to structural performance; University of Coimbra; November 2007

[6] Anderberg, Y.; The impact of various material models on structural fire behaviour prediction; Structures in fire '08; Singapore 2008

[7] Gernay, T.; Franssen J.-M.; A formulation of the Eurocode 2 concrete model at elevated temperature that includes an explicit term for transient creep; Fire Safety Journal 51 (2012) 1 - 9

[8] Gernay, T.; Effect of transient creep strain model on the behaviour of concrete columns subjected to heating and cooling; Fire technology 48 (2012) 313 - 329 
[9] Huang, S.; Burgess, I.; Huang, Z. and Plank, R.; Effect of transient thermal strain on the bucklingof slender concrete and concrete-filled columns in fire; Structures in fire '08; Singapore 2008

[10] Schneider U., Behaviour of Concrete at High Temperatures; Deutshcer Ausschuss für Stahlbeton; Booklet 337; Berlin 1982; ISBN 3-433-00958-9

[11] Bazant, Z.; Kaplan, M.; Concrete at high temperatures - Material properties and mathematical models; Longman Group Limited; London 1996; ISBN 0-582-08626-4

[12] Schneider, U. (Editor); Properties of materials at high temperatures: Concrete; Gesamthochschule Kassel Universität / RILEM 1985; ISBN 3-88122-252-9

[13] EN 1992-1-1:2004 Design of concrete structures, Part 1-1: General rules and rules for buildings

[14] Khoury, G.; Majorana, C.; Pesavento, F. and Schrefler, B.; Modelling of heated concrete; Magazine of concrete research 54 (2002) 77 - 101

[15] Schneider, U.; Behaviour of concrete under thermal steady state and non-steady state conditions; Fire and Materials 1 (1976) 103 - 115

[16] Wang, Y.; Burgess, I.; Wald, F. and Gillie, M.; Performance-based fire engineering of structures; CRC Press; 2013; ISBN 978-0-415-55733-7

[17] Usmani, A.S.; Rotter, J.M.; Lamont, S.; Sanad, A.M.; Gillie, M.; Fundamental principles of structural behaviour under thermal effects; Fire Safety Journal 36 (2001) 721 - 744 